\title{
Black hole mining in the RST model
}

\author{
Rohitvarma Basavaraju* and David A. Lowe† \\ Department of Physics, Brown University, Providence, RI 02912, USA
}

(Dated: October 15, 2018)

\begin{abstract}
We consider the possibility of mining black holes in the 1+1-dimensional dilaton gravity model of Russo, Susskind and Thorlacius. The model correctly incorporates Hawking radiation and backreaction in a semiclassical expansion in $1 / N$, where $N$ is the number of matter species. It is shown that the lifetime of a perturbed black hole is independent of the addition of any extra apparatus when realized by an arbitrary positive energy matter source. We conclude that mining does not occur in the RST model and comment on the implications of this for the black hole information paradox.
\end{abstract}

\footnotetext{
* rohitvarma_basavaraju@brown.edu

$\dagger$ lowe@brown.edu
} 


\section{INTRODUCTION}

Hawking showed that black holes radiate quantum mechanically and eventually evaporate [1]. It is interesting to wonder if there is a consistent manner in which one might perturb a black hole to cause it to evaporate more quickly. Unruh and Wald [2, 3] have argued that radiation from black holes can be mined rapidly, potentially diminishing the lifetime of a 4-dimensional Schwarzschild black hole of mass $M$ to a time of order $M$. The unperturbed lifetime on the other hand is of order $M^{3}$. Such a drastic drop raises thorny questions concerning the black hole information paradox, where apparently one needs at least a scrambling time of order $M \log M$ before quantum information may be carried off by the Hawking radiation [4-7]. By considering optimal semiclassical solutions that accomplish mining, [8] suggests that such a process may at most diminish the lifetime of a black hole to of order $M^{2}$, safely greater than the scrambling time, but parametrically shorter than the unperturbed lifetime.

In the present work our goal is not to test the idea of rapid mining, but simply to consider whether any mining is possible in a model where the question is fully tractable. The original model computation of mining presented in [3] was motivated by a two-dimensional moving mirror in flat spacetime. The models of two-dimensional dilaton gravity provide an ideal testing ground for this particular question, which are a natural generalization of the moving mirror model. In particular, the RST model [9] incorporates a reflecting boundary condition, as well as a full treatment of back-reaction of the Hawking radiation on the geometry at the semiclassical level. Using the scalar matter fields of RST, our strategy will be to send an arbitrary scalar waveform into an evaporating black hole spacetime. We then check if the black hole lifetime decreases. Although there exists a literature on energy conservation in RST [10, 11], the connection with mining and the computation of the lifetime for general flux is new.

\section{REVIEW OF SEMICLASSICAL TWO-DIMENSIONAL DILATON GRAVITY COUPLED TO MATTER IN RST MODEL}

As our basic approach is to consider the effect of arbitrary flux on a black hole in the RST model, we review the relevant elements of that model here. A useful general review of 
these models is [12].

\section{A. Action and Equations of Motion}

The RST action can be thought of as a one loop quantum corrected version of the dilaton gravity action due to Callan, Giddings, Harvey and Strominger (CGHS)[13]:

$$
\begin{aligned}
S & =\frac{1}{2 \pi} \int d^{2} x \sqrt{-g}\left[e^{-2 \phi}\left(R+4(\nabla \phi)^{2}+4 \lambda^{2}\right)-\frac{1}{2} \sum_{i=1}^{N}\left(\nabla f_{i}\right)^{2}\right] \\
& -\frac{\kappa}{8 \pi} \int d^{2} x \sqrt{-g}\left[R \frac{1}{\nabla^{2}} R+2 \phi R\right] .
\end{aligned}
$$

The action for the RST model is given by:

$$
\begin{aligned}
S & =\frac{1}{\pi} \int d^{2} x\left[e^{-2 \phi}\left(2 \partial_{+} \partial_{-} \rho-4 \partial_{+} \phi \partial_{-} \phi+\lambda^{2} e^{2 \rho}\right)\right. \\
& \left.+\frac{1}{2} \sum_{i=1}^{N} \partial_{+} f_{i} \partial_{-} f_{i}-\frac{\kappa}{\pi}\left(\partial_{+} \rho \partial_{-} \rho+\phi \partial_{+} \partial_{-} \rho\right)\right]
\end{aligned}
$$

in conformal gauge where the only non-vanishing components of the metric are $g_{-+}=g_{+-}=$ $-\frac{1}{2} e^{2 \rho} . \phi$ is the dilaton and $f_{i}$ are matter scalar fields. The constants $\kappa=\frac{N \hbar}{12 \pi}$ and $\lambda$ play the roles of Planck's constant and the cosmological constant in this model. We will generally set the latter to unity for convenience. We work in null Kruskal-type coordinates. The $\kappa$ dependent terms in (1) represent the effect of integrating out the one-loop fluctuations of matter fields, as well as the RST improvement term, responsible for maintaining the solubility of the equations of motion. We note the constraint equations in this gauge couple the matter fields to the gravitational fields $\rho$ and $\phi$

$$
\begin{aligned}
0 & =T_{ \pm \pm}=\left(e^{-2 \phi}-\frac{\kappa}{4}\right)\left(4 \partial_{ \pm} \rho \partial_{ \pm} \phi-2 \partial_{ \pm}^{2} \phi\right)+\frac{1}{2} \sum_{i=1}^{N} \partial_{ \pm} f_{i} \partial_{ \pm} f_{i} \\
& -\kappa\left(\partial_{ \pm} \rho \partial_{ \pm} \rho-\partial_{ \pm}^{2} \rho+t_{ \pm}\right) .
\end{aligned}
$$

The functions $t_{ \pm}$are fixed by boundary conditions on the stress energy tensor. We identify the matter contribution to the stress tensor as: 


$$
T_{ \pm \pm}^{f}=\frac{1}{2} \sum_{i=1}^{N} \partial_{ \pm} f_{i} \partial_{ \pm} f_{i} .
$$

The following field redefinitions lead to especially simple equations of motion:

$$
\begin{gathered}
\Omega=\frac{\sqrt{\kappa}}{2} \phi+\frac{e^{-2 \phi}}{\sqrt{\kappa}} \\
\chi=\sqrt{\kappa} \rho-\frac{\sqrt{\kappa}}{2} \phi+\frac{e^{-2 \phi}}{\sqrt{\kappa}} .
\end{gathered}
$$

The resulting action, equations of motion and constraints are:

$$
\begin{gathered}
S=\frac{1}{\pi} \int d^{2} x\left[-\partial_{+} \chi \partial_{-} \chi+\partial_{+} \Omega \partial_{-} \Omega+\lambda^{2} e^{\frac{2}{\sqrt{\kappa}}(\chi-\Omega)}+\frac{1}{2} \sum_{i=1}^{N} \partial_{+} f_{i} \partial_{-} f_{i}\right] \\
\partial_{+} \partial_{-} \chi=\partial_{+} \partial_{-} \Omega=-\frac{\lambda^{2}}{\sqrt{\kappa}} e^{\frac{2}{\sqrt{\kappa}}(\chi-\Omega)} \\
\kappa t_{ \pm}=-\partial_{ \pm} \chi \partial_{ \pm} \chi+\sqrt{\kappa} \partial_{ \pm}^{2} \chi+\partial_{ \pm} \Omega \partial_{ \pm} \Omega+\frac{1}{2} \sum_{i=1}^{N} \partial_{ \pm} f_{i} \partial_{ \pm} f_{i} .
\end{gathered}
$$

We further fix the gauge to Kruskal gauge where $\chi=\Omega$.

\section{B. On Boundaries, Singularities and the Apparent Horizon}

In calculating the effect of flux on the black hole lifetime in RST, we will be making use

of the boundary of the spacetime, the linear dilaton vacuum (LDV), and the positions of the black hole singularity and apparent horizon. We now review these concepts and their relations.

The LDV solution is the analog of higher dimensional flat spacetime and is given by

$$
\Omega=\frac{x^{+} x^{-}}{\sqrt{\kappa}}-\frac{\sqrt{\kappa}}{4} \log \left(-x^{+} x^{-}\right) .
$$

The scalar curvature is given by:

$$
R=\frac{8\left[1-(\nabla \phi)^{2}\right]}{\sqrt{\kappa} e^{2 \phi} \Omega^{\prime}(\phi)} .
$$


This will be generically singular at the critical value $\phi=\phi_{c r}$ where $d \Omega / d \phi=0$ which we denote by the curve $\hat{x}^{+}\left(x^{-}\right)$.

The apparent horizon is the boundary of a trapped region. The variable $e^{-\phi}$ can be thought of as a analog of a transverse sphere in the dimensional reduction of four-dimensional Einstein gravity to two-dimensional dilaton gravity. The quantity $\nabla \phi$ will therefore be timelike in a trapped region. The boundary is determined by the equation $(\nabla \phi)^{2}=0$ which becomes

$$
\partial_{+} \Omega=0
$$

In both subcritical and supercritical regimes, there is a unique curve $\phi=\phi_{c r}$. In the former case, this is the boundary of the LDV. In the latter case, it is the black hole singularity where (4) diverges. To maintain cosmic censorship, RST observe that finite curvature at the boundary requires imposing the cosmic censorship boundary condition

$$
\partial_{ \pm} \Omega=0
$$

This implies reflective boundary conditions for the energy flux

$$
T_{--}^{f}-\kappa t_{-}=\left(\frac{d \widehat{x}^{+}}{d x^{-}}\right)^{2}\left(T_{++}^{f}-\kappa t_{+}\right)
$$

\section{GENERALIZING RST TO ARBITRARY MATTER FLUX}

We review here the reflection of subcritical flux and black hole formation from supercritical flux. These cases constitute the general solutions to RST for arbitrary matter flux in the strictly subcritical and supercritical regimes. We then consider a general black hole solution with general ingoing subcritical flux.

\section{A. Review of Subcritical and Supercritical Cases}

A subcritical flux obeys the following inequality [14]

$$
T_{++}^{f}<\frac{\kappa}{4\left(x^{+}\right)^{2}}
$$

When the flux always obeys this condition the boundary remains timelike and a black hole

does not form. Such flux can be made to reflect from the boundary of the spacetime given 
appropriate boundary conditions as shown in figure 1. The opposite inequality will imply that the boundary curve becomes a spacelike singularity, which is identified as the black hole singularity. We refer to this latter case, where black hole formation occurs, as "supercritical" (see figure 2).

For illustrative purposes, we review the manner in which purely subcritical flux is reflected in RST. In the subsequent section the same procedure will be used to reflect flux outside the event horizon of a black hole. We will assume the ingoing subcritical flux is localized in some region $x_{i}^{+}<x^{+}<x_{f}^{+}$. The Bondi energy $M_{+}\left(x^{+}\right)$and the Kruskal momentum $P_{+}\left(x^{+}\right)$ are defined as integrals along $\mathscr{I}^{-}$

$$
\begin{gathered}
P_{+}\left(x^{+}\right)=\int_{x_{i}^{+}}^{x^{+}} T_{++}^{f} d x^{+} \\
M_{+}\left(x^{+}\right)=\int_{x_{i}^{+}}^{x^{+}} T_{++}^{f} x^{+} d x^{+} .
\end{gathered}
$$

In fact, only these two integrals are needed since in our choice of gauge the flux is related to the double derivative of $\Omega$ :

$$
-\sqrt{\kappa} \partial_{ \pm}^{2} \Omega=-\kappa t_{ \pm}+\frac{1}{2} \sum_{i=1}^{N} \partial_{ \pm} f_{i} \partial_{ \pm} f_{i}
$$

In the case of subcritical flux, one can trace out the reflection of the flux from the boundary as shown in figure 1. There are three regions of interest separated by lines of constant $x^{-}$, namely $x^{-}=x_{i}^{-}$and $x^{-}=x_{f}^{-}$, defined via $x_{i}^{+}=\hat{x}^{+}\left(x_{i}^{-}\right)$and $x_{f}^{+}=\hat{x}^{+}\left(x_{f}^{-}\right)$.

The first region contains only infalling flux:

$$
\Omega^{(i)}\left(x^{-}<x_{i}^{-}\right)=-\frac{x^{+}\left(x^{-}+P_{+}\left(x^{+}\right)\right)}{\sqrt{\kappa}}-\frac{\sqrt{\kappa}}{4} \log \left(-x^{+} x^{-}\right)+\frac{M_{+}\left(x^{+}\right)}{\sqrt{\kappa}} .
$$

The function $t_{+}=\frac{1}{4 x^{+2}}$ is determined by demanding the solution approach the linear dilaton vacuum as $x^{-} \rightarrow-\infty$. The second region corresponds to the reflection of flux, with (6) determining the outgoing flux $T_{--}=T_{--}^{f}-\kappa t_{-}$

$$
\begin{aligned}
\Omega^{(i i)}\left(x_{i}^{-}\right. & \left.<x^{-}<x_{f}^{-}\right)=-\frac{x^{+}\left(x^{-}+P_{+}\left(x^{+}\right)\right)}{\sqrt{\kappa}}-\frac{\sqrt{\kappa}}{4} \log \left(-x^{+} x^{-}\right) \\
& +\frac{M_{+}\left(x^{+}\right)}{\sqrt{\kappa}}+F\left(x^{-}\right)
\end{aligned}
$$




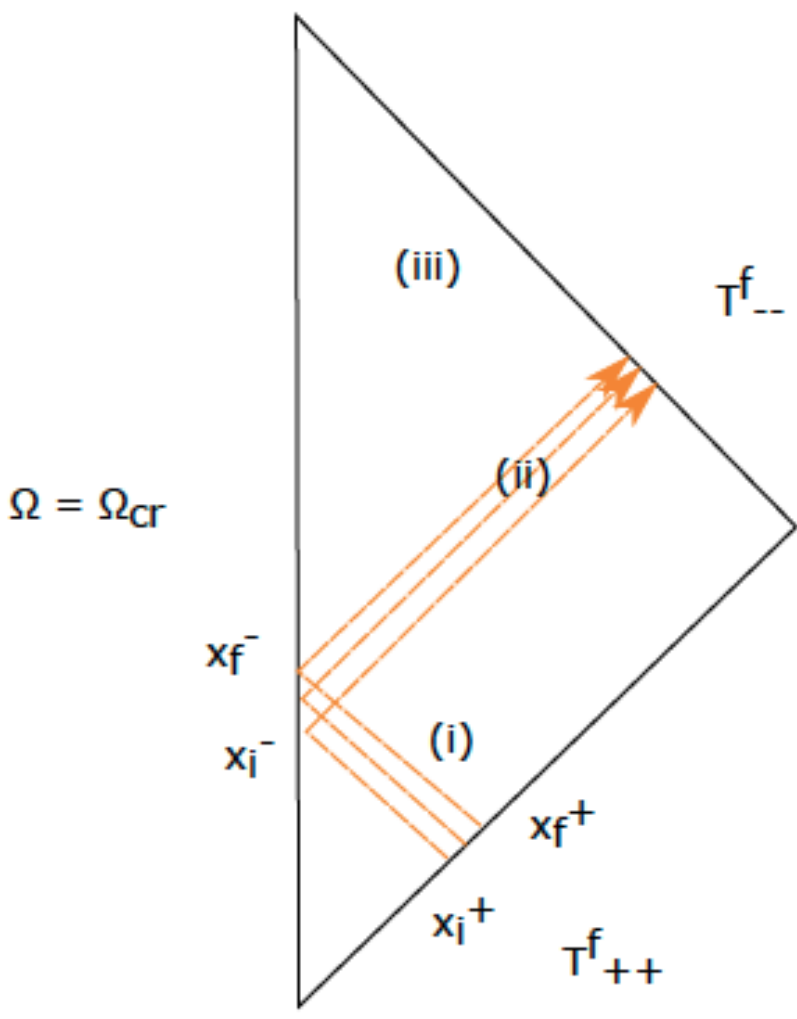

Figure 1. Reflection of subcritical flux in RST with three distinct regions corresponding to infall of flux, reflection and LDV aftermath.

$$
F\left(x^{-}\right)=\frac{\sqrt{\kappa}}{4} \log \left(-x^{-} \hat{x}^{+}\right)-\frac{M\left(\hat{x}^{+}\right)}{\sqrt{\kappa}}-\frac{\sqrt{\kappa}}{4} \log \left(\frac{\kappa}{4}\right)
$$

The function $F\left(x^{-}\right)$and the shape of the boundary curve $\hat{x}^{+}\left(x^{-}\right)$is determined from the finite curvature conditions (5). The third region is the empty linear dilaton vacuum (where we denote the constants $P_{+}^{f} \equiv P\left(x_{f}^{+}\right)$and $\left.M_{+}^{f} \equiv M\left(x_{f}^{+}\right)\right)$:

$$
\Omega^{(i i i)}\left(x^{-}>x_{f}^{-}\right)=-\frac{x^{+}\left(x^{-}+P_{+}^{f}\right)}{\sqrt{\kappa}}-\frac{\sqrt{\kappa}}{4} \log \left(-x^{+}\left(x^{-}+P_{+}^{f}\right)\right) .
$$

Having reviewed the manner in which subcritical flux is reflected with RST boundary conditions, as well as how black hole formation occurs, we now turn to an analysis of a general black hole solution with additional subcritical flux probing the black hole. 


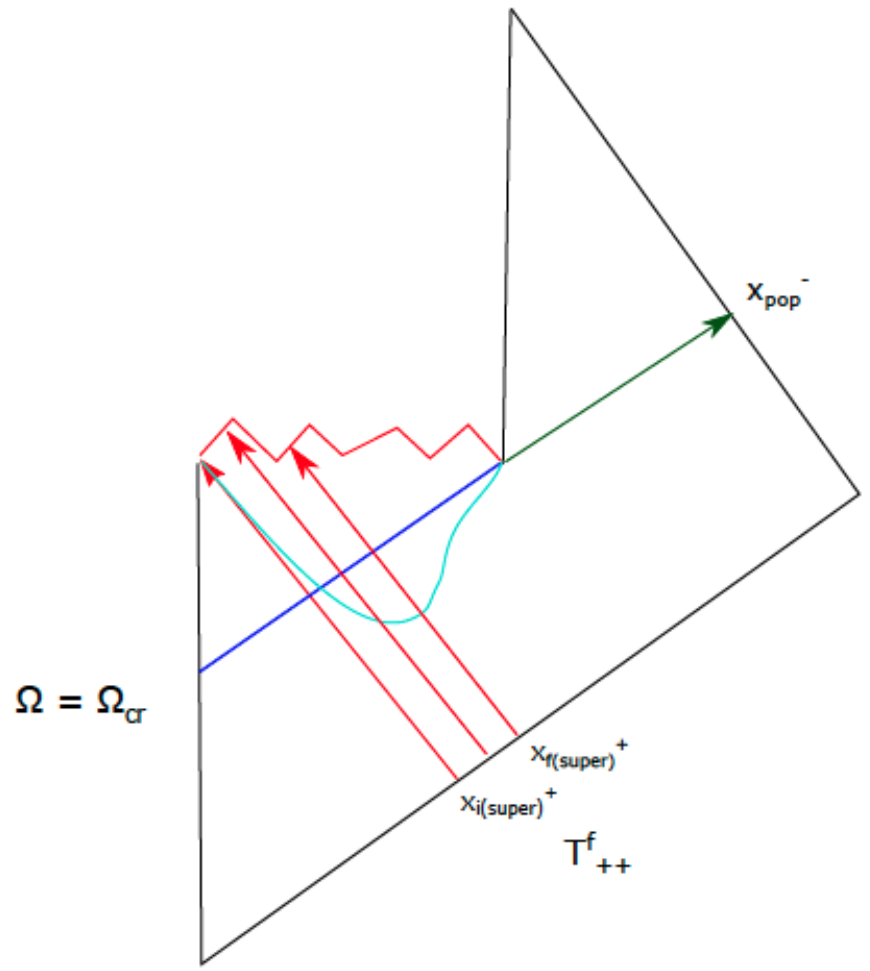

Figure 2. Black hole formation by supercritical flux, shown in red. Event horizon shown in blue, apparent horizon in turquoise, thunderpop in green and black hole singularity in red.

\section{B. General Black Hole Solution and Thunderpop}

The endpoint of Hawking radiation in RST results in a well known emission of energy referred to as the "thunderpop" [14. We take the viewpoint that the thunderpop is to be viewed as a condition to be imposed on the low energy theory to make it semiclassically consistent, representing the effect of some higher derivative interactions that descend from some unknown ultraviolet complete theory. This allows us to avoid the issues raised in 12 where the thunderpop was interpreted as a squeezed state in the semiclassical theory that becomes problematic when evolved back in time. The goal of the present section is to compute the general position of the thunderpop $\left(x_{p o p}^{+}, x_{p o p}^{-}\right)$.

We will take a LDV solution with additional flux sent in from some interval in $x^{+}$on $\mathscr{I}^{-}$corresponding to $x_{i(\text { front })}^{+}<x^{+}<x_{f(\text { behind })}^{+} \leq x_{\text {pop }}^{+}$(see figure 3). We will assume the

flux turns supercritical solely in a sub-interval $x_{i(\text { super })}^{+}<x^{+}<x_{f(\text { super })}^{+}$(see figure 3). The consequent general black hole solution is given by: 


$$
\begin{aligned}
\Omega^{(i)}\left(x^{+}>x_{i(\text { super })}^{+}\right) & =-\frac{x^{+} x^{-}}{\sqrt{\kappa}}-\frac{\sqrt{\kappa}}{4} \log \left(-x^{+} x^{-}\right)+\Omega_{(\text {front })}^{(i)}\left(x^{+}\right)+\Omega_{(\text {behind })}\left(x^{+}\right) \\
& +\Omega_{(\text {super })}\left(x^{+}\right)
\end{aligned}
$$

where the total flux contribution has been separated into separate physically significant pieces. In general, the effect of each piece of flux is of the form:

$$
\delta \Omega\left(x^{+}\right)=-\frac{x^{+}}{\sqrt{\kappa}} \delta P_{+}+\frac{\delta M_{+}}{\sqrt{\kappa}}
$$

where we are permitted to break the contributions into pieces and linearly sum since integrals over some interval can be split and summed over corresponding sub-intervals.

We include a general supercritical contribution $\Omega_{(\text {super })}$ which forms the black hole and we also separate the subcritical flux which is reflected outside the event horizon $\Omega_{(\text {front })}^{(i)}$ from the portion that passes behind $\Omega_{(\text {behind })}$ and propagates to the singularity. As the thunderpop corresponds to a null line crossing the endpoint of black hole evaporation, it can also be taken to define the event horizon (see figures 2 and 3 . We will assume that no flux is sent in after the black hole has evaporated. Further, we will assume that the flux outside the event horizon is reflected in a manner that follows the RST prescription, hence our usage of the superscript $(i)$ on $\Omega_{(\text {front })}^{(i)}$.

The reflected solution $\Omega^{(i i)}$ outside the horizon is given by applying the RST boundary conditions. In the causal past of the thunderpop we have:

$$
\begin{gathered}
\Omega^{(i i)}=-\frac{x^{+}\left(x^{-}+P_{+(\text {front })}\left(x^{+}\right)\right)}{\sqrt{\kappa}}-\frac{\sqrt{\kappa}}{4} \log \left(-x^{+} x^{-}\right)+ \\
\frac{M_{+(\text {front })}\left(x^{+}\right)}{\sqrt{\kappa}}+F_{(\text {front })}\left(x^{-}\right)+\Omega_{B H}\left(x^{+}\right),
\end{gathered}
$$

where we define:

$$
\Omega_{B H} \equiv \Omega_{(\text {behind })}+\Omega_{(\text {super })} .
$$

Evaluating $\Omega^{(i i)}$ in the region to be patched to the evaporated LDV: $x^{+}>x_{\text {pop }}^{+}$as well as along the thunderpop itself: $x^{-}=x_{\text {pop }}^{-}$results in the following simplified form for $\Omega^{(i i)}$ : 


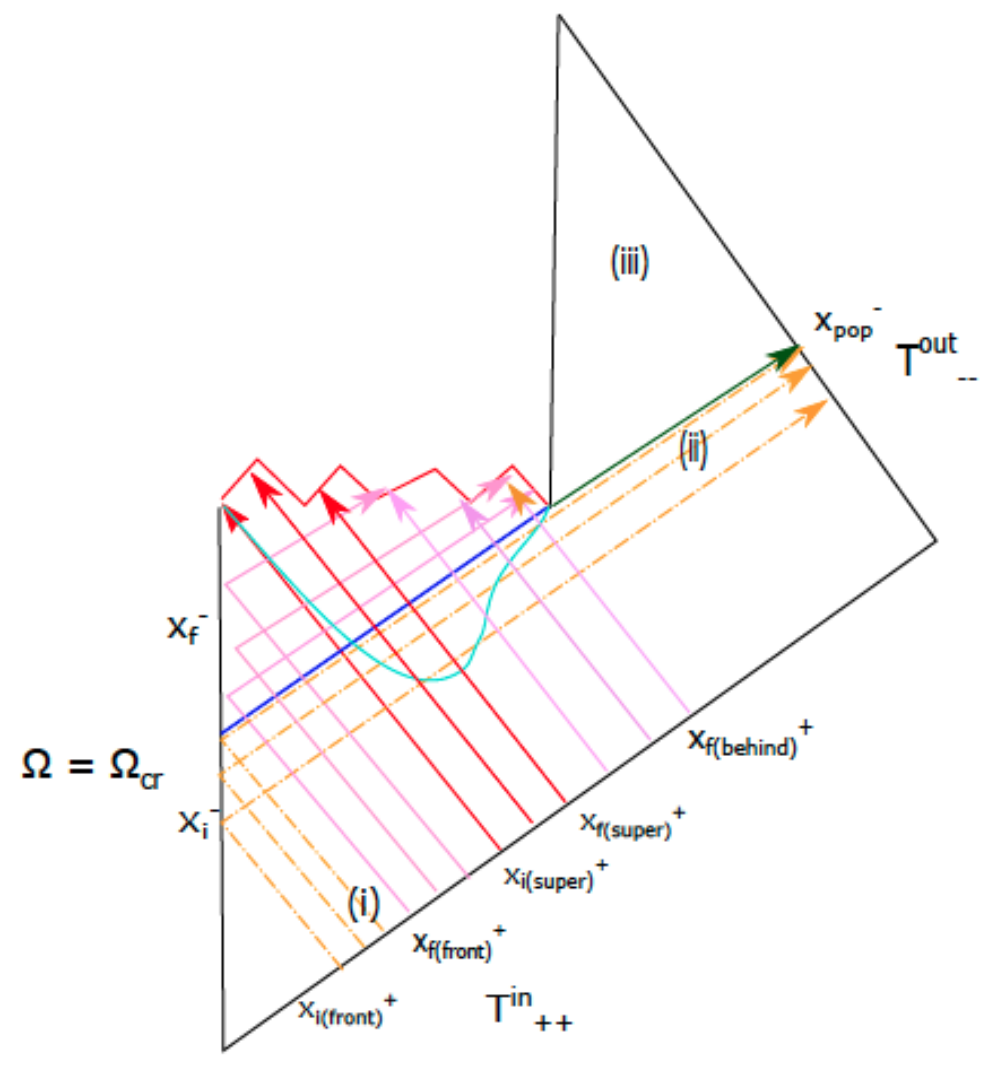

Figure 3. General flux probing general black hole. Subcritical fluxes which remain outside and pass inside the black hole are shown in orange and purple respectively. Supercritical flux is shown in red, the event horizon is shown in blue, and the apparent horizon is shown in turquoise. Regions (i) $\left(x^{-}<x_{i}^{-}\right)$, (ii) $\left(x_{i}^{-}<x^{-}<x_{p o p}^{-}\right)$and (iii) $\left(x_{(p o p)}^{-}<x^{-}\right)$are the natural generalizations from the RST prescription for subcritical reflected flux.

$$
\begin{aligned}
\Omega^{(i i)}\left(x^{+}>x_{\text {pop }}^{+}, x^{-}=x_{\text {pop }}^{-}\right) & =-\frac{x^{+}\left(x_{\text {pop }}^{-}+P_{+(\text {front })}^{f}\right)}{\sqrt{\kappa}} \\
& -\frac{\sqrt{\kappa}}{4} \log \left(-x^{+}\left(x_{\text {pop }}^{-}+P_{+(\text {front })}^{f}\right)\right)+\Omega_{B H}\left(x^{+}\right),
\end{aligned}
$$

which can be easily patched to an appropriately shifted LDV (3)

$$
\begin{aligned}
\Omega^{(i i i)} & =-\frac{x^{+}\left(x_{\text {pop }}^{-}+P_{+(\text {front })}^{f}+P_{+(\text {behind })}^{f}+P_{+(\text {super })}^{f}\right)}{\sqrt{\kappa}} \\
& -\frac{\sqrt{\kappa}}{4} \log \left(-x^{+}\left(x_{\text {pop }}^{-}+P_{+(\text {front })}^{f}+P_{+(\text {behind })}^{f}+P_{+(\text {super })}^{f}\right)\right) .
\end{aligned}
$$


The continuity of such a patching entails the following form of $x_{p o p}^{-}$:

$$
x_{\text {pop }}^{-}=-P_{+(\text {front })}^{f}+\frac{P_{+(\text {behind })}^{f}+P_{+(\text {super })}^{f}}{e^{-4\left(M_{+(\text {super })}^{f}+M_{+(\text {behind })}^{f}\right) / \kappa}-1} .
$$

Using the position for the apparent horizon $\partial_{+} \Omega=0$ on $(8)$ and noting no additional flux is sent in near the endpoint, we obtain

$$
x_{\text {pop }}^{+}=-\frac{\kappa}{4\left(x_{\text {pop }}^{-}+P_{+(\text {front })}^{f}+P_{+(\text {behind })}^{f}+P_{+(\text {super })}^{f}\right)}=\frac{\kappa\left(e^{4\left(M_{+(\text {super })}^{f}+M_{+(\text {behind })}^{f}\right) / \kappa}-1\right)}{4\left(P_{+(\text {super })}^{f}+P_{(\text {behind })}^{f}\right)} .
$$

The determination of this general thunderpop position is the first main technical result of this paper.

\section{MEASURING LIFETIME WITH THE EVENT HORIZON}

The black hole region can be defined as the complement of the causal past of $\mathscr{I}^{+}[15]$, which is bounded in part by the event horizon. We observe that the event horizon ought to be defined by a null line which intersects the thunderpop and is therefore given in the region $x^{+}<x_{\text {pop }}^{+}$by the thunderpop coordinate $x_{E H}^{-}=x_{p o p}^{-}(9)$. The endpoint of evaporation will be given by the thunderpop as both the event horizon and the apparent horizon meet at this point (see figures (2) and (3)). The initial point of black hole formation will be the

projection of the thunderpop coordinate onto the boundary curve, $x_{B H}^{+}=\hat{x}^{+}\left(x_{p o p}^{-}\right)$as this corresponds to the edge of the event horizon. We further project these boundary points of the event horizon onto $\mathscr{I}^{-}$, enabling us to measure the lifetime using the asymptotically flat light-cone coordinate $\sigma^{+}$:

$$
x^{+}=e^{\sigma^{+}}
$$

To determine the lifetime of the black hole, we need to determine where the null line of the event horizon intersects the timelike boundary curve where the RST boundary conditions are applied (5). An equation governing the boundary curve can be obtained by solving $\partial_{+} \Omega=0$ on (7)

$$
\hat{x}^{+}=-\frac{\kappa}{4\left(\hat{x}^{-}+P_{+(\text {front })}\left(\hat{x}^{+}\right)\right)} .
$$


Substituting in for $x_{E H}^{-}$gives

$$
x_{E H}^{+}=\frac{\kappa}{4} \frac{1-e^{-4\left(M_{+(\text {super })}^{f}+M_{+(\text {behind })}^{f}\right) / \kappa}}{P_{+(\text {behind })}^{f}+P_{+(\text {super })}^{f}} .
$$

Converting (10) and (12) to the asymptotically flat coordinate (11), one arrives at the following:

$$
\Delta \sigma^{+}=\log \left(\frac{e^{4\left(M_{+(\text {super })}^{f}+M_{+(\text {behind })}^{f}\right) / \kappa}-1}{1-e^{-4\left(M_{+(\text {super })}^{f}+M_{+(\text {behind })}^{f}\right.}}\right)=\frac{4 M_{\text {tot }}}{\kappa}>\frac{4 M_{+(\text {super })}^{f}}{\kappa},
$$

where the mass of the black hole is defined as

$$
M_{\text {tot }}=M_{+(\text {super })}^{f}+M_{+(\text {behind })}^{f},
$$

which implies that mining does not occur. The determination of this general lifetime is the second main technical result of this paper. The expression is monotonically increasing in the mass and grows arbitrarily large in the classical limit of small $\kappa$. In particular, the lifetime is clearly larger than in the case where probing subcritical flux is absent. Moreover, it is manifest that this lifetime is immune to any flux which remains outside the event horizon and in fact grows in precisely the way one would expect for flux which passes behind the horizon. This shows that in a model where back-reaction is treated in a self-consistent manner, mining does not occur.

\section{CONCLUSIONS}

The issue of mining a black hole has been considered for the first time in a quantum model with back-reaction incorporated at leading order in a $1 / N$ expansion. This provides a useful generalization of the moving mirror model that appearing in the original paper by Unruh and Wald [3], which was used to justify the proposal that energy could be mined from Schwarzschild black holes. We conclude that with quantum back-reaction properly incorporated, no mining takes place in the model we consider.

The general philosophy of this kind of computation is that it correctly captures the qualitative physics of an s-wave reduction of four-dimensional Einstein gravity in the semiclassical limit. By modifying the model, adding in some hopefully harmless extra terms, it can be 
rendered exactly soluble. This then provides motivation to reconsider the mining proposal of Unruh and Wald in the context of such soluble models.

A key feature of the mining proposal is that one should be able to mine modes irrespective of their angular momentum. This is crucial in order to get rapid mining of the type further explored in a later essay by Unruh and Wald [2]. It would be interesting to generalize the considerations of the present paper to this case. A useful soluble model for this, which includes an analog of the potential barrier for such modes is the two-dimensional charged black hole, which has a soluble version. It is also interesting to generalize the cosmic censorship RST boundary conditions considered in the present work to more general energy conserving boundary conditions of the type studied in later dilaton gravity papers [16-18].

The original proposal of Unruh and Wald suggested that black holes can be mined at a rate of a Planck energy per unit Planck time. This would allow a rapidly mined black hole to evaporate in a light crossing time. Such a rapid evaporation poses severe problems for extracting quantum information from black holes, in holographic approaches to quantum gravity. Subsequent work incorporating classical back-reaction led to the minimum mining lifetime being walked back to a time of $O\left(M^{2}\right)$ [8]. The conclusions of the present work support the idea that mining of Schwarzschild black holes cannot occur once quantum backreaction is incorporated, potentially strengthening the conclusions of Brown.

[1] S. W. Hawking, "Particle Creation by Black Holes," Commun. Math. Phys. 43 (1975) 199-220.

[2] W. G. Unruh and R. M. Wald, "How to mine energy from a black hole," General Relativity and Gravitation 15 no. 3, (1983) 195-199. http://dx.doi.org/10.1007/BF00759206.

[3] W. G. Unruh and R. M. Wald, "Acceleration radiation and the generalized second law of thermodynamics," Phys. Rev. D 25 (Feb, 1982) 942-958. http://link.aps.org/doi/10.1103/PhysRevD.25.942

[4] P. Hayden and J. Preskill, "Black holes as mirrors: Quantum information in random subsystems," JHEP 09 (2007) 120, arXiv:0708.4025 [hep-th].

[5] Y. Sekino and L. Susskind, "Fast Scramblers," JHEP 10 (2008) 065, arXiv: 0808.2096 [hep-th]. 
[6] D. A. Lowe and L. Thorlacius, "Quantum information erasure inside black holes," JHEP 12 (2015) 096, arXiv:1508.06572 [hep-th]

[7] D. A. Lowe and L. Thorlacius, "A holographic model for black hole complementarity," arXiv: 1605.02061 [hep-th].

[8] A. R. Brown, "Tensile Strength and the Mining of Black Holes," Phys. Rev. Lett. 111 no. 21, (2013) 211301, arXiv:1207.3342 [gr-qc].

[9] J. G. Russo, L. Susskind, and L. Thorlacius, "Cosmic censorship in two-dimensional gravity," Phys. Rev. D47 (1993) 533-539, arXiv:hep-th/9209012 [hep-th]

[10] W. T. Kim and J. Lee, "ADM, Bondi mass, and energy conservation in two-dimensional dilaton gravities," Int. J. Mod. Phys. A11 (1996) 553-562, arXiv:hep-th/9502078 [hep-th].

[11] W. T. Kim and J. Lee, "Hawking radiation and energy conservation in an evaporating black hole," Phys. Rev. D52 (1995) 2232-2238, arXiv:hep-th/9502115 [hep-th].

[12] A. Strominger, "Les Houches lectures on black holes," in NATO Advanced Study Institute: Les Houches Summer School, Session 62: Fluctuating Geometries in Statistical Mechanics and Field Theory Les Houches, France, August 2-September 9, 1994. 1994. arXiv:hep-th/9501071 [hep-th].

[13] C. G. Callan, Jr., S. B. Giddings, J. A. Harvey, and A. Strominger, "Evanescent black holes," Phys. Rev. D45 no. 4, (1992) R1005, arXiv:hep-th/9111056 [hep-th].

[14] J. G. Russo, L. Susskind, and L. Thorlacius, "The Endpoint of Hawking radiation," Phys. Rev. D46 (1992) 3444-3449, arXiv:hep-th/9206070 [hep-th].

[15] R. M. Wald, General Relativity. The University of Chicago Press, 1984.

[16] T. Chung and H. Verlinde, "Dynamical moving mirrors and black holes," Nuclear Physics B418 (1994) 305-336.

[17] S. R. Das and S. Mukherji, "Boundary dynamics in dilaton gravity," Mod. Phys. Lett. A9 (1994) 3105-3118, arXiv:hep-th/9407015 [hep-th].

[18] A. Strominger and L. Thorlacius, "Conformally invariant boundary conditions for dilaton gravity," Phys. Rev. D50 (1994) 5177-5187, arXiv:hep-th/9405084 [hep-th]. 